\title{
Electrically controlled dc and ac transport in bilayer $\mathrm{WSe}_{2}$
}

\author{
M. Tahir, ${ }^{1, *}$ P. M. Krstajić,,${ }^{2, \dagger}$ and P. Vasilopoulos ${ }^{3, \ddagger}$ \\ ${ }^{1}$ Department of Physics, Colorado State University, Fort Collins, Colorado 80523, USA \\ ${ }^{2}$ Center of Microelectronic Technologies, ICTM, University of Belgrade, Njegoševa 12, 11000 Belgrade, Serbia \\ ${ }^{3}$ Department of Physics, Concordia University, 7141 Sherbrooke Ouest, Montréal, Québec H4B 1R6, Canada
}

(Received 8 December 2017; revised manuscript received 6 June 2018; published 27 August 2018)

\begin{abstract}
We investigate quantum transport in a bilayer $\mathrm{WSe}_{2}$ in the presence of an external potential $V$ and interlayer coupling. The dc and ac conductivities are evaluated in the framework of linear response theory. As functions of the Fermi energy, the dc ones exhibit a nonmonotonic behavior with a flat region in the band gap. A deep local minimum is visible in spin-Hall conductivity near the top of the valence band as a consequence of a large valence band splitting. On the other hand, the ac spin- and valley-Hall conductivities, as functions of the frequency, show two opposite peaks near the top of the valence band. The spin-Hall conductivity exhibits a minimum value at $V=0$ whereas the valley-Hall conductivity decreases monotonically upon varying $V$ from negative to positive values. Furthermore, we evaluate the power absorption spectrum and assess its dependence on the spin and valley degrees of freedom. The results are pertinent to the development of electrically controlled spintronic and valleytronic devices based on bilayer $\mathrm{WSe}_{2}$ and other group VI semiconductors.
\end{abstract}

DOI: 10.1103/PhysRevB.98.075429

\section{INTRODUCTION}

In recent years, a lot of attention has been paid to experimental and theoretical investigations of monolayers of group VI transition metal dichalcogenides (e.g., $M X_{2}, M=\mathrm{Mo}$, $\mathrm{W} ; X=\mathrm{S}, \mathrm{Se}$ ) because of their novel electronic and optical properties arising from their unique band structures [1-6]. This is because $M X_{2}$ monolayers have a hexagonal honeycomb structure similar to that of graphene, but with huge direct band gaps and strong spin-orbit coupling (SOC). The former is due to the breaking of the inversion symmetry and the latter because the heavy metal's $d$ orbitals possess SOC, due to which the spin and valley degrees of freedom are locked and give rise to exotic effects such as the valley-Hall effect $[7,8]$, the spin-Hall effect [9,10], the quantum anomalous Hall effect [11], the valley optical selection rules [2-5], and transistors [12,13].

In addition to $M X_{2}$ monolayers, there has been a lot of interest in $\mathrm{MX}_{2}$ bilayers. Bilayers exhibit rich electronic properties such as electrical polarization [14,15], electrically tuned magnetic moments [16], magnetoelectric effect [17,18], and spin-layer locking physics [19]. Further, $\mathrm{MX}_{2}$ heterostructures (bilayers) are particularly important to photonics and optoelectronics applications because of the strong excitonic effect and valley-dependent phenomena [20-24], such as ultrafast charge transfer $[25,26]$ and formation of interlayer excitons [23,27]. These studies have focused on heterobilayers [22,23,25-29], such as $\mathrm{MoSe}_{2} / \mathrm{WSe}_{2}$ and $\mathrm{MoS}_{2} / \mathrm{WS}_{2}$, in which the built-in interfacial electric field limits significantly the tunability of interlayer excitons. On the other hand, this built-in electric field is absent in pristine homobilayers that are

\footnotetext{
*m.tahir06@alumni.imperial.ac.uk

†predrag222@gmail.com

†p.vasilopoulos@concordia.ca
}

formed by two identical $M X_{2}$ monolayers. The weak interlayer interaction further allows the separation of bilayer systems into monolayer-like components by modest vertical electric fields $[16,19,31-33]$. The earlier experimental studies reported insignificant field effects on intralayer excitons [16,19,33] whereas the application of a vertical electric field in recent [34] high-quality bilayer samples allowed the generation of layerpolarized exciton gases with widely tunable properties and presented new opportunities for tunable electronic, photonic, and optoelectronic devices [22,29,30].

It is important to note that the spin splitting in $M X_{2}$ monolayers disappears almost completely in bilayers. Indeed, in spintronic and valleytronic applications, it may be useful to have a material in which the polarization can be switched on and off. This can be achieved if the inversion symmetry in the bilayer is broken by an external electric field normal to the $M X_{2}$ plane as it leads to a potential difference between the individual layers and allows the control of valley polarization [16]. This effect should be even more pronounced in $\mathrm{WSe}_{2}$ bilayers that show stronger spin-orbit splittings than the $\mathrm{MoS}_{2}$ ones. A $\mathrm{WSe}_{2}$ monolayer possesses a huge band gap $2 \Delta=$ $1.7 \mathrm{eV}$ and large SOC $\left[2 \lambda_{v}^{\prime}=450 \mathrm{meV}, 2 \lambda_{c}^{\prime}=30 \mathrm{meV}\right.$ ], and $\mathrm{WSe}_{2}$ samples are of high quality [34]. In contrast, bilayer graphene has intrinsically a very weak SOC $[35,36]$ and, if not biased, a zero band gap [37,38]. Although this gap can be controlled by an electric field $E_{z}$ [39-42], high-quality samples of $\mathrm{WSe}_{2}$ bilayers with a strong intrinsic SOC and a huge band gap are of particular importance.

In light of the above, we consider an external electric field applied normal to a $\mathrm{WSe}_{2}$ bilayer. This field breaks the inversion symmetry of $M X_{2}$ monolayers, reduces the electronic band gap linearly with its strength, and creates significant spin splittings which quickly reach values similar to those in monolayers. These observations can be explained as a consequence of locking spin and layer pseudospin in a 
given valley [19]. This fact clearly demonstrates the important coupling between spin and layer pseudospin in bilayer $\mathrm{WSe}_{2}$ and opens the possibility of spin and valley manipulation that is important in the fields of spintronics, valleytronics, and quantum logic [18].

More specifically, we investigate dc and ac transport in a bilayer of $\mathrm{WSe}_{2}$ in the presence of interlayer coupling and a perpendicular electric field. After presenting the basics of the model and the linear response conductivities $[43,44]$ in Sec. II, we evaluate the latter numerically in Sec. III. Then in Sec. IV we calculate the power absorption spectrum, assess its dependence on the valley degree of freedom and $V$, and summarize in Sec. V.

\section{FORMALISM}

\section{A. Energy spectrum and eigenfunctions}

A minimal band model of bilayer $\mathrm{WSe}_{2}$ in the neighborhood of the $K$ points can be constructed by adding interlayer coupling to the $\mathbf{k} \cdot \mathbf{p}$ model of monolayers established in Refs. [1619]. In the presence of a perpendicular electric potential $V$, the Hamiltonian takes the form

$$
H=\left(\begin{array}{cccc}
-\xi_{1}^{s \tau} & \text { at } k_{-} & \gamma & 0 \\
a t k_{+} & \xi_{2}^{s \tau} & 0 & 0 \\
\gamma & 0 & -\xi_{3}^{s \tau} & \text { at } k_{+} \\
0 & 0 & \text { at } k_{-} & \xi_{4}^{s \tau}
\end{array}\right),
$$

where $\quad k_{ \pm}=\tau k_{x} \pm i k_{y}, \quad \xi_{1}^{s \tau}=\Delta+V+\tau s \lambda_{v}, \xi_{2}^{s \tau}=\Delta-$ $V-\tau s \lambda_{c}, \xi_{3}^{s \tau}=\Delta-V-\tau s \lambda_{v}$, and $\xi_{4}^{s \tau}=\Delta+V+\tau s \lambda_{c}$. Notice that the term $\Delta$ can be neglected near the band edges; cf. Refs. $[18,19]$. The basis is

$$
\begin{aligned}
& \left\{\left(\left|d_{x^{2}-y^{2}}^{u}\right\rangle-i \tau\left|d_{x y}^{u}\right|\right) / \sqrt{2},\left|d_{z^{2}}^{u}\right\rangle,\right. \\
& \left.\quad\left(\left|d_{x^{2}-y^{2}}^{l}\right\rangle+i \tau\left|d_{x y}^{l}\right|\right) / \sqrt{2},\left|d_{z^{2}}^{l}\right\rangle\right\}^{T},
\end{aligned}
$$

where $T$ denotes the transpose and the superscripts " $\mathrm{u}$ " and "l" denote the upper and lower layers, respectively. Here $\mathbf{k}$ is the relative wave vector with respect to the $K$ points, $\Delta$ is the monolayer band gap, $a$ is the lattice constant, $t$ is the nearest-neighbor intralayer hopping, with $\lambda_{c}, \lambda_{v}$ being the SOC strengths for electrons and holes, and $V$ is the applied electric field energy. Further, $\gamma$ is the interlayer coupling for holes, while the interlayer hopping for electrons vanishes at $K$ points due to the symmetry of the $d_{z^{2}}$ orbital. $\tau= \pm 1$ is the valley index of bilayer bands and $s$ denotes the spin. Similar to monolayers, the spin-up and spin-down states are still decoupled in bilayers, and interlayer coupling conserves spin.

We now discuss the eigenvalues and eigenfunctions of bilayer $\mathrm{WSe}_{2}$ without a magnetic field, near the $K$ and $K^{\prime}$ valleys. Then Eq. (1) can be written in polar form as

$$
H^{\tau}=\left(\begin{array}{cccc}
-\xi_{1}^{s \tau} & \omega \tau e^{-i \tau \theta_{k}} & \gamma & 0 \\
\omega \tau e^{i \tau \theta_{k}} & \xi_{2}^{s \tau} & 0 & 0 \\
\gamma & 0 & -\xi_{3}^{s \tau} & \omega \tau e^{i \tau \theta_{k}} \\
0 & 0 & \omega \tau e^{-i \tau \theta_{k}} & \xi_{4}^{s \tau}
\end{array}\right)
$$

where $\quad \tau k_{x} \pm i k_{y}=k \tau e^{ \pm i \tau \theta_{k}}, \tan \left(\tau \theta_{k}\right)=\tau\left(k_{y} / k_{x}\right), k=$ $\left[k_{x}^{2}+k_{y}^{2}\right]^{1 / 2}, \omega=\hbar v_{F} k$, and $v_{F}=a t$.
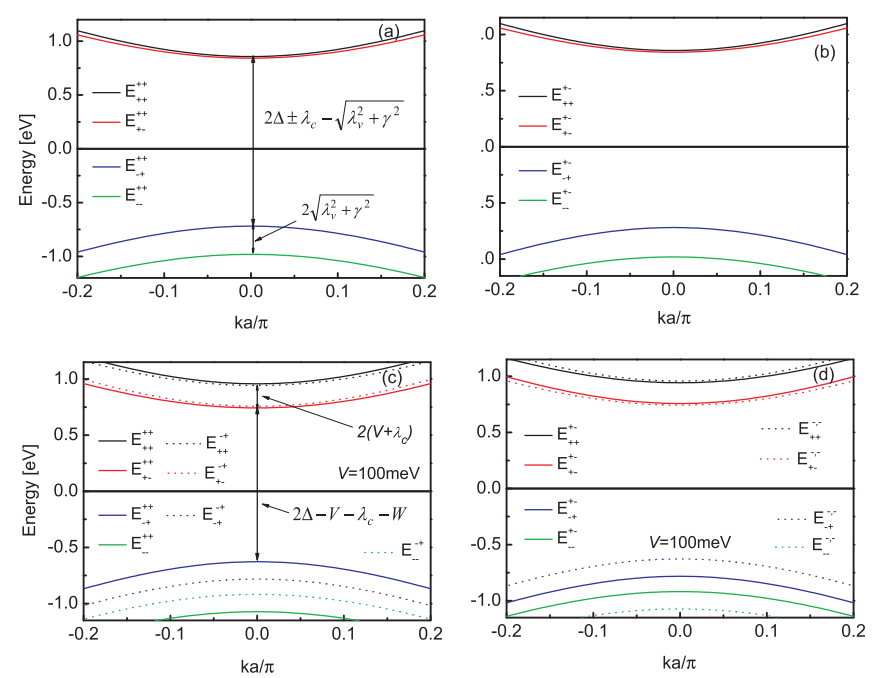

FIG. 1. Energy dispersion for bilayer $\mathrm{WSe}_{2}$ for $\Delta=0.85 \mathrm{eV}$, $\lambda_{c}=7.5 \mathrm{meV}, \lambda_{v}=112.5 \mathrm{meV}$, and $\gamma=0.067 \mathrm{eV}$. The upper panels are for zero electric field energy $V=0$, whereas the lower panels are for $V=100 \mathrm{meV}$. The left (right) panels are for the $K\left(K^{\prime}\right)$ valley and $W=\sqrt{\left(V+\lambda_{v}\right)^{2}+\gamma^{2}}$.

The eigenvalue problem pertinent to Eq. (3) leads to the quartic equation for the energy $E$

$$
E^{4}+a_{1} E^{2}+a_{2} E+a_{3}=0,
$$

where

$a_{1}=-\xi_{1}^{s \tau} \xi_{2}^{s \tau}-\xi_{3}^{s \tau} \xi_{4}^{s \tau}+\left(\xi_{2}^{s \tau}-\xi_{1}^{s \tau}\right)\left(\xi_{4}^{s \tau}-\xi_{3}^{s \tau}\right)-\gamma^{2}-2 \omega^{2}$,

$a_{2}=\left(\xi_{2}^{s \tau}-\xi_{1}^{s \tau}\right) \xi_{3}^{s \tau} \xi_{4}^{s \tau}+\left(\xi_{4}^{s \tau}-\xi_{3}^{s \tau}\right) \xi_{1}^{s \tau} \xi_{2}^{s \tau}+\gamma^{2}\left(\xi_{2}^{s \tau}+\xi_{4}^{s \tau}\right)$

$$
a_{3}=\xi_{1}^{s \tau} \xi_{2}^{s \tau} \xi_{3}^{s \tau} \xi_{4}^{s \tau}+\omega^{2}\left(\xi_{1}^{s \tau} \xi_{2}^{s \tau}+\xi_{3}^{s \tau} \xi_{4}^{s \tau}\right)-\gamma^{2} \xi_{2}^{s \tau} \xi_{4}^{s \tau}+\omega^{4}
$$

The solutions of Eq. (3) are

$$
\begin{aligned}
E_{\mu_{1} \mu_{2}}^{s, \tau}= & \frac{1}{2 \sqrt{3}}\left\{\mu_{1}\left[-2 a_{1}+B+A / 2^{1 / 3}\right]^{1 / 2}\right. \\
& \left.+\mu_{2}\left[4 a_{1}+B+\frac{6 \sqrt{3} a_{2} \mu_{1}}{\sqrt{-2 a_{1}+3 B+A / 2^{1 / 3}}}\right]^{1 / 2}\right\},
\end{aligned}
$$

where $B=2^{1 / 3}\left(a_{1}^{2}+12 a_{3}\right) / A$ and

$$
A=\left[C+\sqrt{-4\left(a_{1}^{2}+12 a_{3}\right)^{2}+C^{2}}\right]^{\frac{1}{3}},
$$

with $C=2 a_{1}^{3}+27 a_{2}^{2}-72 a_{1} a_{3}$. Here $\mu_{1}=+1(-1)$ is for the conduction (valence) band whereas $\mu_{2}=+1(-1)$ is for the upper (lower) layer.

In the upper panels of Fig. 1, we show the energy dispersion for bilayer $\mathrm{WSe}_{2}$, at zero electric field $E_{z}=0(V=0 \mathrm{meV})$. We note the following: (i) The splitting due to the SOC in the conduction band is negligible and (ii) the splitting due to interlayer coupling in the conduction band is zero but finite in 
the valence band. Furthermore, the splitting of the valence band comes as a result of the combined effect of interlay coupling and SOC and is given by $2 \sqrt{\lambda_{v}^{2}+\gamma^{2}}$ at $\mathbf{k}=0$. This implies that the valence band is split even for $\lambda_{v}=0$. (iii) The gap between the conduction and valence band edges is approximately given by $2 \Delta-\sqrt{\lambda_{v}^{2}+\gamma^{2}}$. In the lower panels of Fig. 1, we show the energy dispersion for a finite electric field $E_{z}(V=100 \mathrm{meV})$. The dotted curves correspond to the spin-down states. We point out the following:

(a) The SOC splitting is modified by the presence of $E_{z}$, while the spin splitting in the conduction band due to SOC is small compared to that of the valence band.

(b) The gap is given by $2 \Delta-V-\lambda_{c}-W$ with $W=$ $\sqrt{\left(V+\lambda_{v}\right)^{2}+\gamma^{2}}$. The gap of monolayer $\mathrm{WSe}_{2}$ is $2 \Delta=$ $1.7 \mathrm{eV}$, the spin-orbit parameters are $\lambda_{c}=7.5 \mathrm{meV}$ and $\lambda_{c}=$ $112.5 \mathrm{meV}$, and the coupling $\gamma=0.067 \mathrm{eV}$ [18]. Note that some bands are degenerate due to the factor $\tau s$ in the diagonal elements $\xi_{i}$ of the Hamiltonian; this entails $E_{\mu_{1} \mu_{2}}^{++}=E_{\mu_{1} \mu_{2}}^{--}$ and $E_{\mu_{1} \mu_{2}}^{+-}=E_{\mu_{1} \mu_{2}}^{-+}$. Notice that the valley and spin degrees of freedom are coupled with the layer pseudospin $[18,19]$.

(c) The spin and layer splittings are enhanced by the

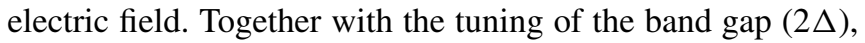
this should be useful in electrically controlled spintronic and valleytronic devices based on bilayer $\mathrm{WSe}_{2}$ and similar group VI semiconductors.

The normalized eigenfunctions corresponding to Eq. (3) are

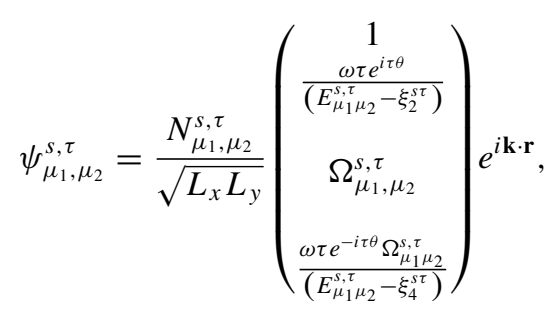

with $\quad \Omega_{\mu_{1} \mu_{2}}^{s, \tau}=\left[\left(E_{\mu_{1} \mu_{2}}^{s, \tau}+\xi_{1}^{s \tau}\right)\left(E_{\mu_{1} \mu_{2}}^{s, \tau}-\xi_{2}^{s \tau}\right)-\omega^{2}\right] /$ $\left[\gamma\left(E_{\mu_{1} \mu_{2}}^{s, \tau}-\xi_{2}^{s \tau}\right)\right]$ and the normalization coefficient

$$
\begin{aligned}
N_{\mu_{1}, \mu_{2}}^{s, \tau}= & \left\{\left(\Omega_{\mu_{1}, \mu_{2}}^{s, \tau}\right)^{2}\left[1+\omega^{2} /\left(E_{\mu_{1} \mu_{2}}^{s, \tau}-\xi_{4}^{s \tau}\right)^{2}\right]\right. \\
& \left.+1+\omega^{2} /\left(E_{\mu_{1} \mu_{2}}^{s, \tau}-\xi_{2}^{s \tau}\right)^{2}\right\}^{-1 / 2}
\end{aligned}
$$

In Fig. 2, we show the density of states (DOS) of bilayer $\mathrm{WSe}_{2}$ as a function of the normalized energy $E / \Delta$ for $V=0$ (black solid curve) and $V=100 \mathrm{meV}$ (red dashed curve). There is a visible asymmetry between the conduction and valence band, which can also be deduced from Fig. 1: The valence band splitting is significantly larger than that of the conduction band. On the other hand, for large energies, the DOS is approximately a linear function of the energy. It can be seen that for $V>0$ (red dashed curve) the gap is reduced by $V+\sqrt{V^{2}+\lambda_{v}^{2}}$. Further, for finite $V$ one sees additional splittings, near the band edges, tied to the spectrum of Figs. 1(c) and 1(d). Together with the spin and layer splitting enhancements by the electric field, these results significantly affect the dc and ac transport properties; see Secs. III and IV.

\section{B. Conductivities}

To determine the conductivities we adopt the formalism of Ref. [43]. We consider a many body-system described

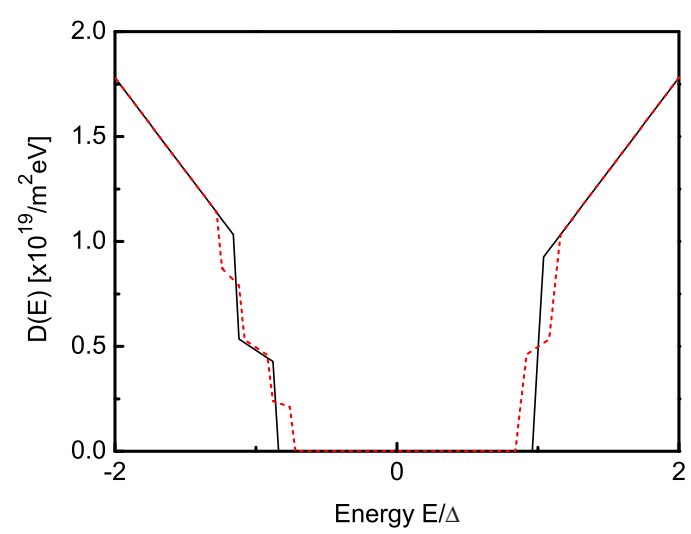

FIG. 2. Density of states $D(E)$ as a function of normalized energy $E / \Delta$ for field energy $V=0$ (solid black curve) and $V=100 \mathrm{meV}$ (red dashed curve).

by the Hamiltonian $H=H_{0}+H_{I}-\mathbf{R} \cdot \mathbf{F}$, where $H_{0}$ is the unperturbed part, $H_{I}$ is a binary type interaction, and $-\mathbf{R} \cdot \mathbf{F}$ is the interaction of the system with an external field $\mathbf{F}$ with $\mathbf{R}=\sum_{i} \mathbf{r}_{i}$ and $\mathbf{r}_{i}$ being the position operator of electron $i$. In the case of interest, $\mathbf{F}=e \mathbf{E}$, where $\mathbf{E}$ is the electric field and $e$ is the electron charge. In the representation in which $H_{0}$ is diagonal, the many-body density operator $\rho$ has a diagonal part $\rho^{d}$ and nondiagonal part $\rho^{n d}, \rho=\rho^{d}+\rho^{n d}$. For weak electric fields and weak scattering, the conductivity tensor has a diagonal and a nondiagonal part, $\sigma_{\mu \nu}=\sigma_{\mu \nu}^{d}+\sigma_{\mu \nu}^{n d}$, $\mu, v=x, y$.

There are two kinds of currents, diffusive and hopping, but usually only one of them is present. When no magnetic field is present, the hopping contribution vanishes identically; see Eq. (2.65) in Ref. [43]. For elastic scattering, the component $\sigma_{\mu \nu}^{d}$ is given by [43]

$$
\sigma_{\mu \nu}^{d}(i \omega)=\frac{\beta e^{2}}{S} \sum_{\zeta} f_{\zeta}\left(1-f_{\zeta}\right) \frac{v_{\nu \zeta} v_{\mu \zeta} \tau_{\zeta}}{1+i \omega \tau_{\zeta}},
$$

where $\omega$ is the frequency, $\tau_{\zeta}$ is the relaxation time, and $v_{\mu \zeta}$ is the diagonal matrix element of the velocity operator, $v_{\mu \zeta}=$ $\left\langle\zeta\left|v_{\mu}\right| \zeta\right\rangle(\mu=x, y)$ with $|\zeta\rangle=\left|\mu_{1}, \mu_{2}, \tau, s_{z}, \mathbf{k}\right\rangle$, and $S$ is the area of the system. Further, $f_{\zeta}$ is the Fermi-Dirac distribution $f_{\zeta}=\left\{1+\exp \left[\beta\left(E_{\zeta}-E_{F}\right)\right]\right\}^{-1}, \beta=1 / k_{B} T$, and $T$ is the temperature.

As far as the contribution $\sigma_{\mu \nu}^{n d}$ is concerned, one can use the identity $f_{\zeta^{\prime}}\left(1-f_{\zeta}\right)\left[1-e^{\beta\left(E_{\zeta^{\prime}}-E_{\zeta}\right)}\right]=f_{\zeta^{\prime}}-f_{\zeta}$ and cast the original expression [43] in the familiar form

$$
\sigma_{\mu \nu}^{n d}(i \omega)=\frac{i \hbar e^{2}}{S} \sum_{\zeta \neq \zeta^{\prime}} \frac{\left(f_{\zeta}-f_{\zeta^{\prime}}\right) v_{\nu \zeta \zeta^{\prime}} v_{\mu \zeta^{\prime} \zeta}}{\left(E_{\zeta}-E_{\zeta^{\prime}}\right)\left(E_{\zeta}-E_{\zeta^{\prime}}+\hbar \omega-i \eta\right)},
$$

where $v_{v \zeta \zeta^{\prime}}=\left\langle\zeta\left|v_{v}\right| \zeta^{\prime}\right\rangle$ and $v_{\mu \zeta^{\prime} \zeta}=\left\langle\zeta^{\prime}\left|v_{\mu}\right| \zeta\right\rangle$ are the offdiagonal matrix elements of the velocity operators with $v, \mu=$ $x, y$. The sum runs over all quantum states $|\zeta\rangle$ and $\left|\zeta^{\prime}\right\rangle$ provided $|\zeta\rangle \neq\left|\zeta^{\prime}\right\rangle$. From now on, the infinitesimal quantity $\eta$ in Eq. (13) will be replaced by $\Gamma_{\zeta}$ in order to account for the finite broadening of the energy levels. 


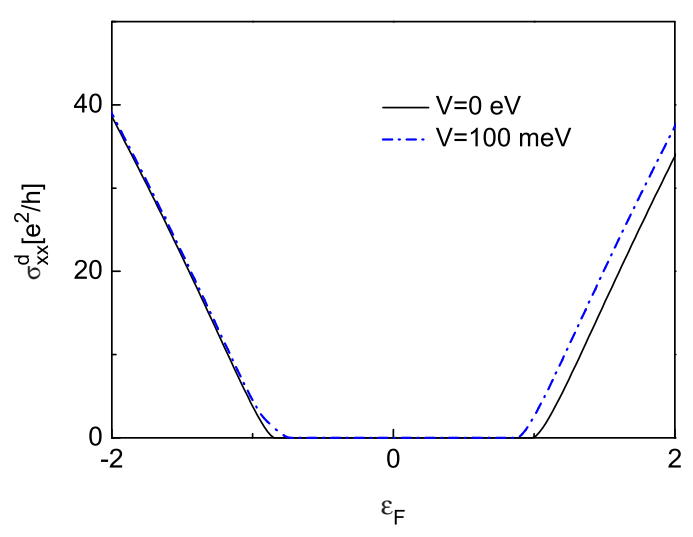

FIG. 3. The diagonal conductivity $\sigma_{x x}^{d}$ vs normalized Fermi energy $\epsilon_{F}=E_{F} / \Delta$ for $\tau_{F}=10^{-14} \mathrm{~s}$ [45]. The black solid curve is for $V=0$ and the blue dash-dotted one for $V=100 \mathrm{meV}$.

\section{NUMERICAL RESULTS}

\section{A. de transport}

First, we present numerical results for the diagonal part $\sigma_{x x}^{d}$ [Eq. (12)] in Fig. 3 as a function of the normalized Fermi energy $\epsilon_{F}=E_{F} / \Delta$ assuming a relaxation time $\tau_{F}=10^{-14} \mathrm{~s}[45]$ and $T=300 \mathrm{~K}$.

It can be seen that the conductivity $\sigma_{x x}^{d}$ decreases monotonically when the Fermi level $E_{F}$ is in the valence band, then it vanishes for $E_{F}$ in the band gap since there are no states to contribute to it, and finally rises again when $E_{F}$ is in the conduction band.

As for the component $\sigma_{x x}^{n d}$, we do not show it because for $\omega=0$ and $\mu=v$ one can interchange the indices $\zeta$ and $\zeta^{\prime}$ in Eq. (13) and take half the sum. Then one finds $\sigma_{x x}^{n d}(0)=0$ for $\eta=0$ or $\sigma_{x x}^{n d}(0) \ll \sigma_{x x}^{d}(0)$ for $\eta \approx 0$.

We now determine the dc spin- and valley-Hall conductivities by setting $\omega=0$ in Eq. (13). As mentioned above in Eq. (12), $\sigma_{y x}^{d}=0$; see Ref. [43]. The results for $\sigma_{y x}^{n d}$ are

$$
\sigma_{y x}^{(s)}=\sum_{\tau}\left[\sigma_{y x}^{n d}\left(\tau, s_{z}=+1\right)-\sigma_{y x}^{n d}\left(\tau, s_{z}=-1\right)\right]
$$

and

$$
\sigma_{y x}^{(v)}=\sum_{s_{z}}\left[\sigma_{y x}^{n d}\left(\tau=+1, s_{z}\right)-\sigma_{y x}^{n d}\left(\tau=-1, s_{z}\right)\right] .
$$

Note that the charge conductivity $\sigma_{y x}^{c}=\sum_{\tau, s_{z}} \sigma_{y x}^{n d}\left(\tau, s_{z}\right)$ vanishes. Since a spin current is defined by $\mathbf{J}_{s}=\hbar /(2 e)\left(\mathbf{J}_{\uparrow}-\mathbf{J}_{\downarrow}\right)$, one has to multiply $\sigma_{y x}^{(s)}$ by $\hbar / 2 e$ and $\sigma_{y x}^{(v)}$ by $1 / 2 e$.

In Figs. 4(a) and 4(b), we plot the spin- and valley-Hall conductivities vs the normalized Fermi energy $\epsilon_{F}=E_{F} / \Delta$. The flat regions centered at $\epsilon_{F}=0$ are due to the fact that factor $f_{+, \mathbf{k}}-f_{-, \mathbf{k}}$ is constant when the Fermi energy lies in a gap, e.g., in the range $-0.75 \leqslant \epsilon_{F} \leqslant 0.85$ in the spin-Hall conductivity for $V=0$; see Fig. 4(a). This occurs because the electric field and the spin current transform in the same way under time-reversal and inversion symmetries. Notice though that the valley-Hall conductivity vanishes in the entire range of $\epsilon_{F}$ used for $V=0$; see Fig. 4(b).

We now inspect the dependence of the spin- and valley-Hall conductivities on the applied electric potential $V$. As shown in
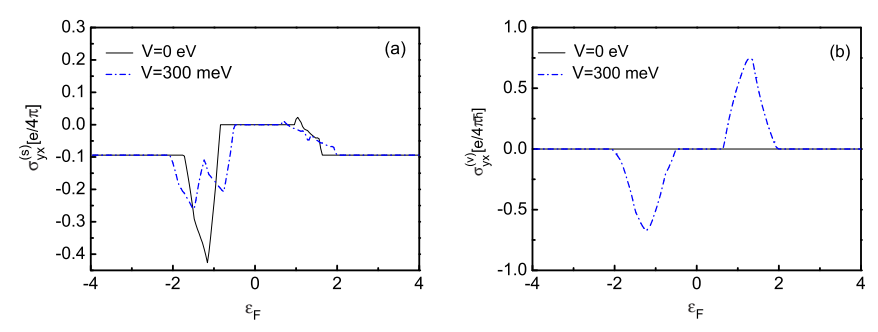

FIG. 4. (a) Spin-Hall conductivity vs normalized Fermi energy at $T=300 \mathrm{~K}$ for $V=0$ (solid curve) and $V=300 \mathrm{meV}$ (dash-dotted curve). (b) As in panel (a) for the valley-Hall conductivity.

Figs. 5(a) and 5(b), the former has a minimum for $V=0$, while the latter decreases with $V$. Furthermore, although not shown, we also studied their behavior for interlayer coupling $\gamma=0$. Neglecting $\gamma$ leads to larger values of the spin-Hall conductivity, especially in the gap, while the trend is the opposite for the valley-Hall conductivity. We remark at this point that a consensus on the value of $\gamma$ has not been reached: Ref. [18] reported the value $\gamma=67 \mathrm{meV}$ determined from $a b$ initio studies, while Ref. [19] used a value that is two orders of magnitudes smaller.

\section{B. ac transport}

In this section, we evaluate the spin- and valley-Hall conductivities for finite frequency, level broadening, and temperature. We assume that the level broadening $\Gamma_{\zeta}$ is the same for all levels, i.e., $\Gamma_{\zeta} \approx \Gamma$.

In Fig. 6(a), we show the diagonal part of the ac conductivity $\sigma_{x x}^{d}(\omega)$, given by Eq. (12), and in Fig. 6(b) is the non-diagonal part, $\sigma_{x x}^{n d}(i \omega)$, obtained from Eq. (13). It can be seen that $\sigma_{x x}^{d}$ monotonically decreases in agreement with the denominator in Eq. (12), while in Fig. 6(b) $\sigma_{x x}^{n d}(\omega)$ exhibits a kink near $\alpha_{0}=2.2$ and a maximum at larger $\alpha_{0}=2.5$ as a result of a large valence band splitting. This behavior is due to the interlayer coupling, huge band gap, SOC, and the onset of interband transitions, which occur, for $V=0$, at $\alpha_{0}=2.2$ and $\alpha_{0}=2.5$, and are associated with bands minima in Fig. 1. Upon increasing $V$, the kink near $\alpha_{0}=3.3$ is further separated and the band gap is tuned (blue dash-dotted curve).

Further, in Fig. 7(a) we show the spin-Hall conductivity, while in Fig. 7(b) we show the valley-Hall conductivity vs normalized frequency $\alpha_{0}=\hbar \omega / \Delta$. The solid black curve is for broadening $\Gamma=20 \mathrm{meV}$ and the red dashed one for $\Gamma=$ $50 \mathrm{meV}$. The Fermi energy is taken to be $E_{F}=0 \mathrm{eV}$ and the
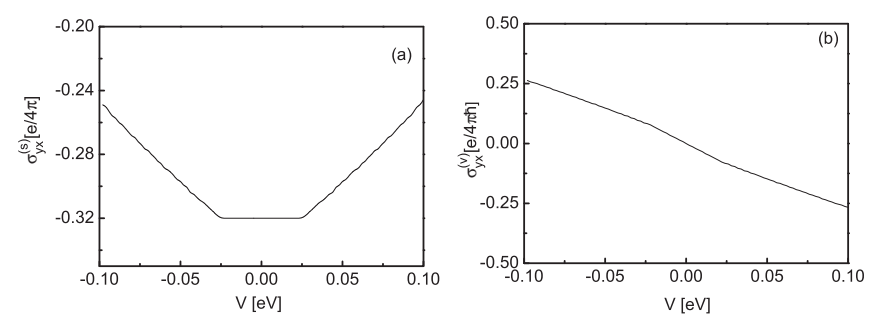

FIG. 5. (a) Spin-Hall conductivity vs potential $V$ for $T=300 \mathrm{~K}$. (b) As in panel (a) for the valley-Hall conductivity. The Fermi level is $E_{F}=-1 \mathrm{eV}$ and the interlayer hopping $\gamma=67 \mathrm{meV}$. 

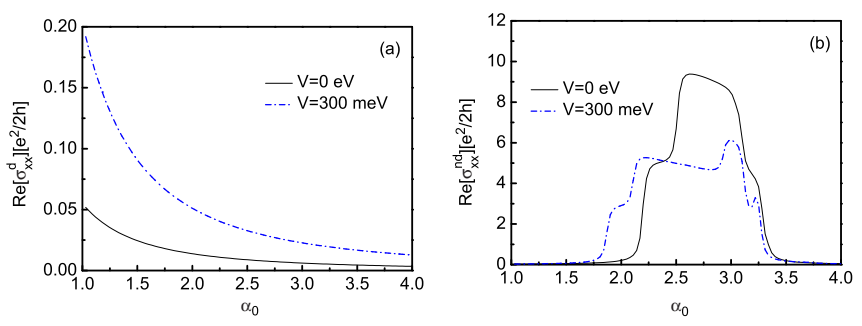

FIG. 6. Diagonal (a) and nondiagonal part (b) of the ac conductivity $\sigma_{x x}(i \omega)$ vs the normalized frequency $\alpha_{0}$. The solid black curve is for $V=0$ and the blue dash-dotted one is for $V=300 \mathrm{meV}$. The parameters used are $E_{F}=1 \mathrm{eV}, \Gamma=20 \mathrm{meV}$, and $\gamma=67 \mathrm{meV}$.

temperature $T=300 \mathrm{~K}$. The double peak near $\alpha_{0}=2$ is due to the large splitting of the valence band and the fact that the conductivities attain large values whenever the denominator in Eq. (13) approaches zero, that is, when $\hbar \omega \approx E_{c}-E_{v} \approx$ $2 \Delta$, i.e., $\alpha_{0} \approx 2$ at $V=0$ (black solid curve). The asymmetric peaks, for $V=0$, are due to the interlayer coupling and SOC; the peak near $1.9 \alpha_{0}$ is due to net spin-up (-down) accumulation in one transverse direction while a negative conductivity yields a net spin-up (-down) accumulation in the opposite direction at $\alpha_{0}=2.2$. It is also visible that a larger level broadening, here $\Gamma=50 \mathrm{meV}$, smears out the peaks.
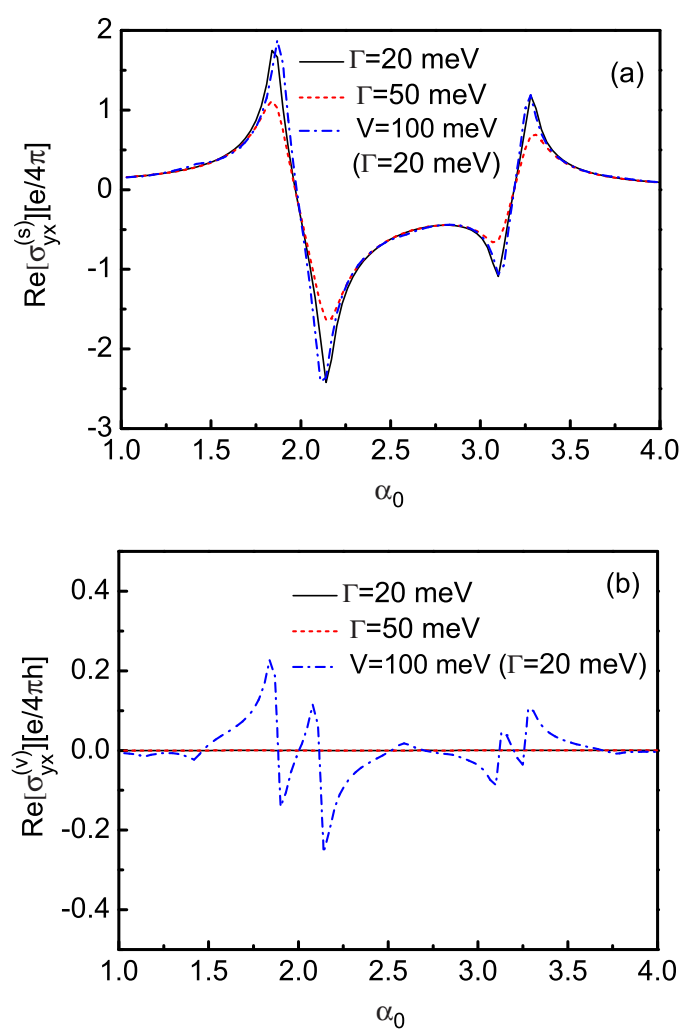

FIG. 7. (a) Spin-Hall conductivity vs frequency for $T=300 \mathrm{~K}$, $E_{F}=0$, and $V=0$. The black solid curves are for $\Gamma=20 \mathrm{meV}$ and the red dashed ones for $\Gamma=50 \mathrm{meV}$. (b) As in panel (a) for the valleyHall conductivity. The blue dash-dotted curve is for $V=100 \mathrm{meV}$ and $\Gamma=20 \mathrm{meV}$.
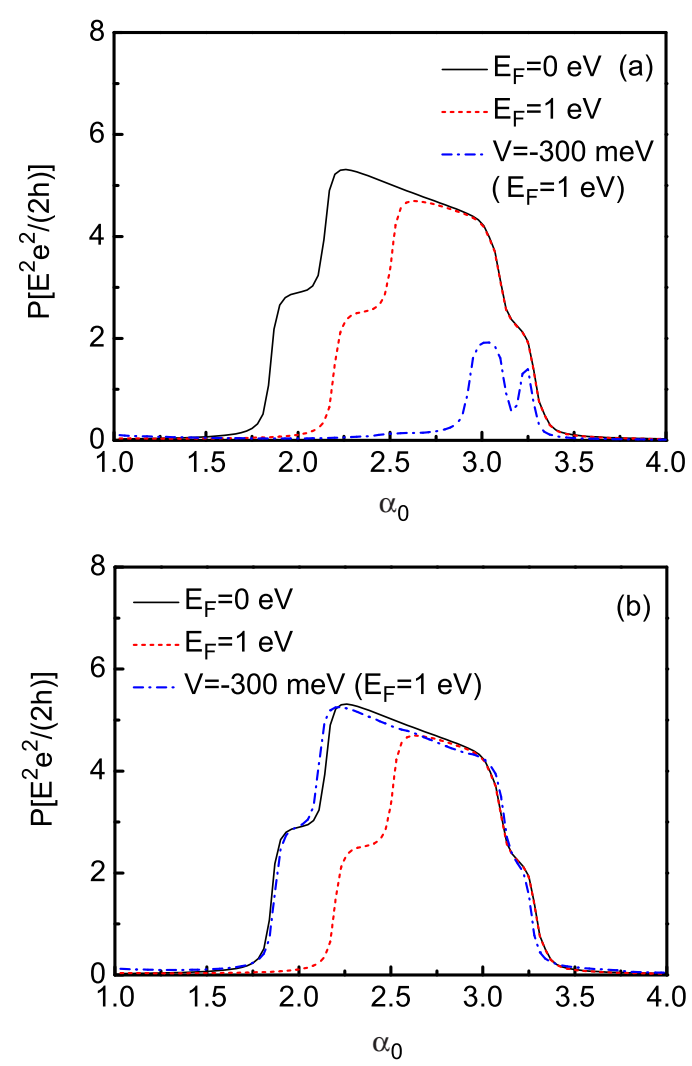

FIG. 8. Power spectrum vs $\alpha_{0}=\hbar \omega / \Delta$ in units of $E^{2} e^{2} / 2 h$ for (a) the $K$ valley and (b) the $K^{\prime}$ valley. In both cases, we used $\tau_{F}=10^{-14} \mathrm{~s}$ [45], $V=0$, and $\Gamma=20 \mathrm{meV}$. The solid black curve corresponds to $E_{F}=0$, the red short-dashed one to $E_{F}=1 \mathrm{eV}$, and the blue dashdotted curve to $V=-300 \mathrm{meV}\left(E_{F}=1 \mathrm{eV}\right)$.

\section{POWER SPECTRUM}

The average power absorbed from circularly polarized light of frequency $\omega$ and electric field $E$ (within linear response theory) is given by

$$
\begin{aligned}
P\left(\omega, \tau, s_{z}\right)= & \left(E^{2} / 2\right) \operatorname{Re}\left\{\sigma_{x x}\left(i \omega, \tau, s_{z}\right)+\sigma_{y y}\left(i \omega, \tau, s_{z}\right)\right. \\
& \left.-i \sigma_{x y}\left(i \omega, \tau, s_{z}\right)+i \sigma_{y x}\left(i \omega, \tau, s_{z}\right)\right\} .
\end{aligned}
$$

We point out that $\sigma_{x x}\left(i \omega, \tau, s_{z}\right)=\sigma_{y y}\left(i \omega, \tau, s_{z}\right)$ and $\sigma_{x y}^{n d}\left(i \omega, \tau, s_{z}\right)=-\sigma_{y x}^{n d}\left(i \omega, \tau, s_{z}\right)$. Further, we have $\sigma_{y x}^{d}\left(i \omega, \tau, s_{z}\right)=-\sigma_{x y}^{d}\left(i \omega, \tau, s_{z}\right)=0$; see Ref. [43]. Then Eq. (16) simplifies to

$$
\begin{aligned}
P\left(\omega, \tau, s_{z}\right)= & E^{2} \operatorname{Re}\left\{\sigma_{x x}^{d}\left(i \omega, \tau, s_{z}\right)\right. \\
& \left.+\sigma_{x x}^{n d}\left(i \omega, \tau, s_{z}\right)+i \sigma_{y x}^{n d}\left(i \omega, \tau, s_{z}\right)\right\} .
\end{aligned}
$$

We consider the two valleys separately but sum over both spin directions. Thus, with $L=K, K^{\prime}$, one may write

$$
P(\omega, L)=P(\omega, L, 1)+P(\omega, L,-1) .
$$

In Figs. 8(a) and 8(b), we show the power spectrum as a function of the normalized frequency $\alpha_{0}$ for the $K$ and $K^{\prime}$ valley, respectively. The common parameters: relaxation time at the Fermi level $\tau_{F}=10^{-14} \mathrm{~s}[45]$ and level broadening $\Gamma=$ $20 \mathrm{meV}$. Further, $E_{F}=0 \mathrm{eV}$ for the solid black and $E_{F}=1 \mathrm{eV}$ for the red short-dashed curve and the blue dash-dotted one 

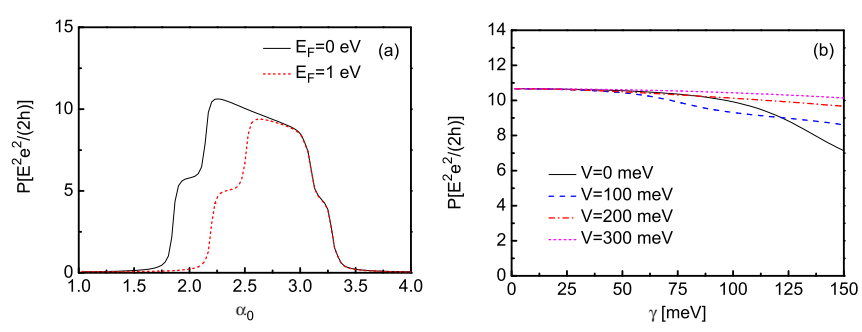

FIG. 9. (a) Power spectrum vs $\alpha_{0}$ in units of $E^{2} e^{2} / 2 h$. The parameters used are $\tau_{F}=10^{-14} \mathrm{~s}[45], V=0, \Gamma=20 \mathrm{meV}$, and $E_{F}=0$ (solid black curve) and $E_{F}=1 \mathrm{eV}$ (red short dashed). (b) Power spectrum vs $\gamma$ for four potentials $V$, at fixed $\alpha_{0}=2.2$ corresponding to the maximum in panel (a).

( $V=-300 \mathrm{meV})$. It can be seen that the values of $P(\omega, K)$ are approximately the same as those of $P\left(\omega, K^{\prime}\right)$ for $V=0 \mathrm{eV}$. One also sees that for large Fermi energy, $E_{F}=1 \mathrm{eV}$ (red short-dashed curve), the kink and maximum are shifted toward higher frequencies. Also, in line with Figs. 6 and 7, the nonmonotonic behavior appears as a result of a large valence band splitting at $V=0$ (black solid curve). The electrostatic potential $V=-300 \mathrm{meV}$ suppresses the contribution of the $K$ valley (blue dash-dotted curve), while for the $K^{\prime}$ valley it shifts to lower frequencies. Though not shown, the influence of the electrostatic potential has minor effects when the Fermi level lies in the middle of the band gap $\left(E_{F}=0 \mathrm{eV}\right)$. In accordance with Figs. 6 and 7, the kink and the maximum in Fig. 8 are due to the interlayer coupling, huge band gap, and SOC. Furthermore, they result from interband transitions which occur at smaller (2.2) $\alpha_{0}$ and larger (2.5) $\alpha_{0}$ and are associated with bands minima in Fig. 1. Upon increasing $V$, the band gap is tuned (blue dash-dotted curve).

The total power spectrum $P(\omega)$ is shown in Fig. 9(a). As in the case of its particular conductivity components, we observe a kink and a maximum, at $\alpha_{0}=2.2$, for $E_{F}=0$ which are shifted to the right for $E_{F}=1 \mathrm{eV}$ (red short-dashed curve). Upon comparing with our similar work on $\mathrm{MoS}_{2}$ (Ref. [46]) we see that in $\mathrm{MoS}_{2}$ we observed a dip between $\alpha_{0}=0$ and $\alpha_{0}=2$, whereas here we have a dip until $\alpha_{0}=1.75$. This can be explained by the facts that (1) the valence band splitting is larger in $\mathrm{WSe}_{2}$ than in $\mathrm{MoS}_{2}$. (2) The conduction band splitting is finite here. In accordance with Figs. 6-8, the nonmonotonic behavior here is due to the interlayer coupling, huge band gap, and SOC. These peaks are due to the onset of interband transitions which occur at $\alpha_{0}=2.2$ and are associated with bands minima shown in Fig. 1. Upon increasing $\mathrm{V}$ the band gap is tuned (blue dash-dotted curve).
Finally, in Fig. 9(b) we show the total power spectrum versus interlayer coupling $\gamma$ for four values of the electric potential: $V=0 \mathrm{meV}$ (solid black curve), $V=100 \mathrm{meV}$ (blue dash-dotted curve), $V=200 \mathrm{meV}$ (red dash-dotted curve), and $V=300 \mathrm{meV}$ (magenta short-dashed curve), all at fixed normalized frequency $\alpha_{0}=2.2$ corresponding to the maximum in Fig. 9(a). We show it as a function of continuous $\gamma$ for pedagogical purposes since a consensus on the value of $\gamma$ has not been reached in the literature. As seen, the power spectrum decreases weakly with $\gamma$. The influence of the potential $V$ is, in general, to weaken this decline.

\section{SUMMARY}

We investigated dc and ac transport in a bilayer of $\mathrm{WSe}_{2}$ in the presence of interlayer coupling and of a perpendicular electric field or potential $V$. The dc and ac conductivities were evaluated numerically using general linear response expressions. The dc spin- and valley-Hall conductivities exhibit a nonmonotonic behavior as functions of the Fermi energy with a visible flat region in the band gap. A large valence band splitting results in two peaks near the top of the valence band for finite $V$. On the other hand, the ac spin- and valley-Hall conductivities, as functions of the frequency, show double peaks near the top of the valence band.

We also evaluated the power absorption spectrum and assessed its dependence on the frequency $\omega$, the valley degree of freedom, and partly the potential $V$. We found out that both valleys contribute equally to the power spectrum $P(\omega)$ when $V=0 \mathrm{eV}$ [see Figs. 8(a) and 8(b)]; this applies to the total power spectrum $P(\omega)$ shown in Fig. 9 as well. Moreover, both $P(\omega, K)$ and $P\left(\omega, K^{\prime}\right)$ have a dip at $\alpha_{0}=\hbar \omega / \Delta=1.75$ In either case, this is tied to the contribution $\sigma_{y x}^{n d}(\omega)$ and the fact that the valence band is split significantly for all values of $\mathbf{k}$.

Our results for $\mathrm{WSe}_{2}$ can be applied to bilayer $\mathrm{MoS}_{2}$ in the limit of zero spin-orbit coupling in the conduction band; cf. Ref. [47]. They can also be extended to other transitionmetal dichalcogenides of the group VI. As such, they have potential applications in electrically controlled spintronic and valleytronic devices based on these materials given that electric knobs have recently been realized that control nanoelectronic devices; see Ref. [48].

\section{ACKNOWLEDGMENTS}

This work was supported by the Colorado State University (M.T.), the Ministry of Education, Science, and Technological Development of Serbia within the Project No. TR 32008 (P.K.), and the Canadian NSERC Grant No. OGP0121756 (P.V.).
[1] D. Xiao, G. B. Liu, W. Feng, X. Xu, and W. Yao, Phys. Rev. Lett. 108, 196802 (2012).

[2] G. Sallen, L. Bouet, X. Marie, G. Wang, C. R. Zhu, W. P. Han, Y. Lu, P. H. Tan, T. Amand, B. L. Liu, and B. Urbaszek, Phys. Rev. B 86, 081301 (2012).

[3] K. F. Mak, K. He, J. Sahn, and T. F. Heinz, Nat. Nanotechnol. 7, 494 (2012).
[4] H. Zeng, J. Dai, W. Yao, D. Xiao, and X. Cui, Nat. Nanotechnol. 7, 490 (2012).

[5] T. Cao, G. Wang, W. Han, H. Ye, C. Zhu, J. Shi, Q. Niu, P. Tan, E. Wang, B. Liu, and J. Feng, Nat. Commun. 3, 887 (2012).

[6] X. Xu, W. Yao, D. Xiao, and T. F. Heinz, Nat. Phys. 10, 343 (2014). 
[7] K. F. Mak, K. L. McGill, J. Park, and P. L. McEuen, Science 344, 1489 (2014).

[8] K. F. Mak and J. Shan, Nat. Photon. 10, 216 (2016).

[9] W. Feng, Y. Yao, W. Zhu, J. Zhou, W. Yao, and D. Xiao, Phys. Rev. B 86, 165108 (2012).

[10] W.-Y. Shan, H.-Z. Lu, and D. Xiao, Phys. Rev. B 88, 125301 (2013).

[11] T. Habe and M. Koshino, Phys. Rev. B 96, 085411 (2017).

[12] H. Fang, S. Chuang, T. C. Chang, K. Takei, T. Takahashi, and A. Javey, Nano Lett. 12, 3788 (2012).

[13] H. C. P. Movva, A. Rai, S. Kang, K. Kim, B. Fallahazad, T. Taniguchi, K. Watanabe, E. Tutuc, and S. K. Banerjee, ACS Nano 9, 10402 (2015).

[14] W. J. Zhao, R. M. Ribeiro, M. L. Toh, A. Carvalho, C. Kloc, A. H. C. Neto, and G. Eda, Nano Lett. 13, 5627 (2013).

[15] B. R. Zhu, H. L. Zeng, J. F. Dai, Z. R. Gong, and X. D. Cui, Proc. Natl. Acad. Sci. USA 111, 11606 (2014).

[16] S. Wu, J. S. Ross, G. B. Liu, G. Aivazian, A. Jones, Z. Y. Fei, W. G. Zhu, D. Xiao, W. Yao, D. Cobden, and X. D. Xu, Nat. Phys. 9, 149 (2013).

[17] H. T. Yuan, M. S. Bahramy, K. Morimoto, S. F. Wu, K. Nomura, B. J. Yang, H. Shimotani, R. Suzuki, M. Toh, C. Kloc, X. D. Xu, R. Arita, N. Nagaosa, and Y. Iwasa, Nat. Phys. 9, 563 (2013).

[18] Z. Gong, G. B. Liu, H. Yu, D. Xiao, X. D. Cui, X. D. Xu, and W. Yao, Nat. Commun. 4, 2053 (2013).

[19] A. M. Jones, H. Yu, J. S. Ross, P. Klement, N. J. Ghimire, J. Q. Yan, D. G. Mandrus, W. Yao, and X. D. Xu, Nat. Phys. 10, 130 (2014).

[20] H. Yu, Y. Wang, Q. Tong, X. Xu, and W. Yao, Phys. Rev. Lett. 115, 187002 (2015).

[21] Y.-M. Li, J. Li, L.-K. Shi, D. Zhang, W. Yang, and K. Chang, Phys. Rev. Lett. 115, 166804 (2015).

[22] J. R. Schaibley, H. Yu, G. Clark, P. Rivera, J. S. Ross, K. L. Seyler, W. Yao, and X. Xu, Nat. Rev. Mater. 1, 16055 (2016).

[23] P. Rivera, K. L. Seyler, H. Yu, J. R. Schaibley, J. Yan, D. G. Mandrus, W. Yao, and X. Xu, Science 351, 688 (2016).

[24] F. Wu, T. Lovorn, and A. H. MacDonald, Phys. Rev. Lett. 118, 147401 (2017).

[25] X. Hong, J. Kim, S.-F. Shi, Y. Zhang, C. Jin, Y. Sun, S. Tongay, J. Wu, Y. Zhang, and F. Wang, Nat. Nano 9, 682 (2014).

[26] F. Ceballos, M.-G. Ju, S. D. Lane, X. C. Zeng, and H. Zhao, Nano Lett. 17, 1623 (2017).

[27] P. Rivera, J. R. Schaibley, A. M. Jones, J. S. Ross, S. Wu, G. Aivazian, P. Klement, K. Seyler, G. Clark, N. J. Ghimire, J. Yan, D. G. Mandrus, W. Yao, and X. Xu, Nat. Commun. 6, 6242 (2015).
[28] J. Kim, C. Jin, B. Chen, H. Cai, T. Zhao, P. Lee, S. Kahn, K. Watanabe, T. Taniguchi, S. Tongay, M. F. Crommie, and F. Wang, arXiv:1612.05359.

[29] C.-H. Lee, G.-H. Lee, A. M. van der Zande, W. Chen, Y. Li, M. Han, X. Cui, G. Arefe, C. Nuckolls, T. F. Heinz, J. Guo, J. Hone, and P. Kim, Nat. Nanotechnol. 9, 676 (2014).

[30] F. Withers, O. D. P. Zamudio, A. Mishchenko, A. P. Rooney, A. Gholinia, K. Watanabe, T. Taniguchi, S. J. Haigh, A. K. Geim, A. I. Tartakovskii, and K. S. Novoselov, Nat. Mater. 14, 301 (2015).

[31] A. Ramasubramaniam, D. Naveh, and E. Towe, Phys. Rev. B 84, 205325 (2011).

[32] J. Lee, K. F. Mak, and J. Shan, Nat. Nano 11, 421 (2016).

[33] J. Klein, J. Wierzbowski, A. Regler, J. Becker, F. Heimbach, K. Müller, M. Kaniber, and J. J. Finley, Nano Lett. 16, 1554 (2016).

[34] Z. Wang, Y.-H. Chiu, K. Honz, K. F. Mak, and J. Shan, arXiv:1612.05359.

[35] F. Guinea, New J. Phys. 12, 083063 (2010).

[36] F. Mireles and J. Schliemann, New J. Phys. 14, 093026 (2012).

[37] M. Zarenia, P. Vasilopoulos, and F. M. Peeters, Phys. Rev. B 85, 245426 (2012).

[38] M. Nakamura, L. Hirasawa, and K. I. Imura, Phys. Rev. B 78, 033403 (2008).

[39] E. McCann, Phys. Rev. B 74, 161403 (2006).

[40] Y. Zhang, T.-T. Tang, C. Girit, Z. Hao, M. C. Martin, A. Zettl, M. F. Crommie, Y. R. Shen, and F. Wang, Nature (London) 459, 820 (2009).

[41] T. Ohta, A. Bostwick, T. Seyller, K. Horn, and E. Rotenberg, Science 313, 951 (2006).

[42] F. Xia, D. B. Farmer, Y. Lin, and P. Avouris, Nano Lett. 10, 715 (2010).

[43] M. Charbonneau, K. M. Van Vliet, and P. Vasilopoulos, J. Math. Phys. 23, 318 (1982).

[44] V. Vargiamidis, P. Vasilopoulos, and G.-Q. Hai, J. Phys.: Condens. Matter 26, 345303 (2014).

[45] Xu Cui, G.-H. Lee, Y. D. Kim, G. Arefe, P. Y. Huang, C.-H. Lee, D. A. Chenet, X. Zhang, L. Wang, F. Ye, F. Pizzocchero, B. S. Jessen, K. Watanabe, T. Taniguchi, D. A. Muller, T. Low, P. Kim, and J. Hone, Nat. Nanotechnol. 10, 534 (2015); H. Schmidt, I. Yudhistira, L. Chu, A. H. Castro Neto, B. Özyilmaz, S. Adam, and G. Eda, Phys. Rev. Lett. 116, 046803 (2016).

[46] P. M. Krstajić, P. Vasilopoulos, and M. Tahir, Phys. Rev. B 94, 085413 (2016).

[47] M. Zubair, M. Tahir, P. Vasilopoulos, and K. Sabeeh, Phys. Rev. B 96, 045405 (2017).

[48] B. Deng, V. Tran, Y. Xie, H. Jiang, C. Li, Q. Guo, X. Wang, H. Tian, S. J. Koester, H. Wang, J. J. Cha, Q. Xia, L. Yang, and F. Xia, Nat. Commun. 8, 14474 (2017). 